\title{
OBSERVATORIOS DE CONVIVENCIA Y SEGURIDAD CIUDADANA: HERRAMIENTAS PARA LA TOMA DE DECISIONES Y GOBERNABILIDAD
}

\author{
Gabriela Sánchez-Rentería ${ }^{1,2, a}$, Francisco Javier Bonilla-Escobar ${ }^{1,3, b}$, Andrés Fandiño-Losada ${ }^{1,4, c}$, \\ María Isabel Gutiérrez-Martinez ${ }^{1,4, d}$
}

\begin{abstract}
RESUMEN
La necesidad de gobernabilidad para generar desarrollo en los países, se ha venido convirtiendo en foco de interés de los gobiernos. América Latina cuenta con sistemas políticos en crisis de gobernabilidad, dada la incapacidad de los actores estratégicos para resolver los conflictos basándose en reglas y procedimientos establecidos. La presente revisión tiene como objetivo mostrar cómo la creación de sistemas de vigilancia en violencia (observatorios) contribuye al fortalecimiento de la gobernabilidad y a la generación de políticas públicas efectivas. Se realizó un análisis de los componentes necesarios para la existencia de gobernabilidad y su relación con el papel que cumplen los observatorios para proporcionar información confiable, oportuna y representativa. La gobernabilidad se enriquece con la legitimidad otorgada por la ciudadanía a partir de los resultados de los gobiernos, en la formulación, implementación, evaluación de políticas públicas y la toma de decisiones en salud pública basadas en evidencia.
\end{abstract}

Palabras clave: Políticas públicas; Vigilancia; Violencia (fuente: DeCS BIREME).

\section{CITIZEN SECURITY OBSERVATORIES: TOOLS FOR DECISION MAKING AND GOVERNABILITY}

\begin{abstract}
The need for good governability to promote countries development has been becoming the focus of governments. Latin America has political systems in crisis of governability caused by the inability of strategic actors to solve conflicts based on rules and procedures. The present review aims to describe how the creation of violence surveillance systems (observatories) contributes to strengthening governability and the creation of effective public policies. It was developed an analysis of the required components for the existence of governability and its relationship with the role of observatories in cities to provide reliable, timely and representative information that allows the formulation of strategies and policies. Governability is enriched with the legitimacy granted by the public from the results obtained by the governments in the formulation, implementation, evaluation of public policies and the evidence-based decisions in public health.
\end{abstract}

Keywords: Public policies; Surveillance; Violence (source: MESH NLM).

\section{INTRODUCCIÓN}

Los eventos de violencia, entendida como el uso deliberado de la fuerza física, en un grado de amenaza o efectivo, contra uno mismo, otra persona, grupo - comunidad, que cause o pueda causar lesiones, muerte o daños psicológicos y la criminalidad como una expresión de la violencia (1), son comunes en las sociedades latinoamericanas. La última década ha estado marcada por un crecimiento y difusión de los delitos, con cifras de hasta un $11 \%$ entre 2000 y 2010 , que provocaron más de un millón de fallecimientos en Latinoamérica y el Caribe por causa de la violencia criminal ${ }^{(2,3)}$.

Cuando los Estados no son capaces de generar desarrollo y los ciudadanos lo perciben, trae consigo desgobierno, economía criminal, violencia, inseguridad y corrupción, empezando a afectar todas las esferas de la vida pública y privada ${ }^{(4)}$. En este contexto, las acciones

\footnotetext{
Instituto Cisalva, Universidad del Valle. Cali, Colombia.

Pontificia Universidad Javeriana de Cali. Cali, Colombia.

Division of Trauma and Surgery, School of Medicine, University of Pittsburgh. Pensilvania, EE.UU.

Escuela de Salud Pública, Universidad del Valle. Cali, Colombia.

Politóloga; ${ }^{\mathrm{b}}$ médico, magíster en Epidemiología; ' médico, doctor en Ciencias Médicas d médica, doctora en Salud Mental y Epidemiología Psiquiátrica Recibido: 06/12/2015 Aprobado: 20/04/2016
} 
estatales se han visto limitadas en la búsqueda por reducir los efectos provocados por problemas sociales y políticos, incapacidad que ha desencadenado la pérdida de su legitimidad y gobernabilidad.

La legitimidad (política), entendida como la confianza, apoyo, consentimiento y aceptación de los individuos sobre un orden político y la acción de las instituciones públicas ${ }^{(5)}$, es un prerrequisito de la gobernabilidad democrática ${ }^{(6)}$. La gobernabilidad es definida como el equilibrio entre las demandas de la sociedad y la capacidad del gobierno para responderlas (7); un sistema social es gobernable cuando está estructurado sociopolíticamente, de modo tal que todos los actores estratégicos se interrelacionan para tomar decisiones colectivas y resolver sus conflictos conforme a un sistema de reglas y de procedimientos formales, o informales, que pueden registrar diversos niveles de institucionalización dentro del cual formulan sus expectativas y estrategias ${ }^{(4)}$.

Cuando los gobiernos no actúan de esta forma, cuando los actores estratégicos se guían por intereses particulares y al no trabajar de forma coordinada, se debilita la gobernabilidad hasta llegar a una crisis, aquel estado de desequilibrio, donde existe una gran cantidad de demandas de la sociedad y poca capacidad de los gobiernos para responderlas, generando, a su vez, un estancamiento en el desarrollo, pues es indispensable que exista gobernabilidad para que se dé su génesis ${ }^{(4,5)}$.

La comunidad cumple un papel trascendental en el ámbito público y social. Es sobre quien recaen tanto los actos violentos como las acciones del Estado para contrarrestarlos. El Estado tiene la responsabilidad directa de eliminar cualquier forma de violencia externa. Cuando sus necesidades no se ven satisfechas y se tiene una percepción de inseguridad, se genera falta de confianza en las instituciones públicas (2). Como consecuencia, se presenta poca colaboración con las autoridades y se deslegitiman las acciones del Estado para hacer frente a la violencia y la criminalidad; puesto que el Estado es un conjunto de instituciones que dentro de un determinado territorio mantiene con éxito el monopolio legítimo de la violencia en pro del mantenimiento del orden vigente ${ }^{(8)}$.

Por lo tanto, es importante lograr un trabajo interinstitucional que permita proveer a la sociedad de acciones legítimas que respondan a realidades políticas y sociales complejas aportando soluciones a problemas que afectan el desarrollo y, a su vez, aumentando el grado de gobernabilidad ${ }^{(9)}$. Uno de los mecanismos que han resultado efectivos para lograr lo anterior son los observatorios de violencia, creados para identificar el problema y generar acciones de prevención en lo local y regional. Se fundamentan en la cooperación interinstitucional para compartir información que permite el análisis de las medidas de seguridad existentes y focalizar las acciones generando políticas públicas y programas de desarrollo que respondan a la evidencia dada por los datos ${ }^{(10,11)}$.

La presente revisión de la literatura tiene como objetivo mostrar cómo la creación de observatorios de violencia contribuye al fortalecimiento de la gobernabilidad y a la generación de políticas públicas efectivas.

\section{LOS OBSERVATORIOS DE VIOLENCIA COMO HERRAMIENTA PARA LA TOMA DE DECISIONES}

En la actualidad, los gobiernos se ven obligados a contar con información confiable, eficiente y oportuna para la toma de decisiones, no solo para resolver situaciones coyunturales sino también para generar políticas públicas que les permitan prevenir situaciones futuras. Estas situaciones son definidas en la agenda pública y son el conjunto de situaciones percibidas que requieren de debate público la intervención de las autoridades públicas ${ }^{(12)}$.

Entonces, para resolver situaciones de manera adecuada, es necesario contar con fuentes de información que permitan realizar un análisis de la situación y sus efectos para tomar decisiones oportunas. A partir de esa necesidad, surgen los observatorios del crimen y la violencia propuesto por el Instituto de Investigación y Desarrollo en Prevención de Violencia y Promoción de la Convivencia Social-CISALVA, donde se permite la caracterización y monitoreo de diferentes eventos de salud, y se promovieron, inicialmente, en torno a lesiones de causa externa, homicidios, suicidios, muertes en eventos de tránsito y muertes por lesiones no intencionales ${ }^{(13)}$. En estos, siguiendo unas etapas definidas se garantiza la obtención de datos confiables y oportunos.

El proceso comienza con la realización de un comité operativo, el cual está conformado por fuentes primarias como la policía, medicina legal, secretaria de tránsito, fiscalía, secretaria de salud y cualquier otra fuente que cuente con la información necesaria que permita el acercamiento a los hechos. En un segundo momento se debe llevar a cabo la sistematización de los datos, análisis estadísticos y georreferenciación para avanzar al comité de análisis, donde se evalúan y contextualizan los resultados obtenidos. Posteriormente, se pasa al comité de política social donde se deben tomar las decisiones consideradas como las más adecuadas para disminuir los índices de violencia y criminalidad.

Se considera que el comité social es el de mayor impacto, pues permite evaluar medidas y estrategias implementadas, y convertir la información en políticas 
públicas. Por último, es necesario que la información se difunda a otras instituciones y a la comunidad incentivando la creación de vínculos de comunicación que permitan generar iniciativas de seguridad comunitaria desde la ciudadanía y mejorar la calidad de la información obtenida ${ }^{(13)}$.

Las acciones que se emprenden a partir del análisis de los datos proporcionados por los observatorios son el resultado de la articulación entre lo político, lo administrativo, lo institucional y lo técnico, que de manera coordinada han logrado llegar a acuerdos que permiten fortalecer las capacidades institucionales, la respuesta oportuna a las necesidades y el acompañamiento técnico a los entes territoriales para optimizar el uso de la información y de los recursos ${ }^{(13)}$

Las instituciones del Estado, los gobiernos locales y los observatorios, actúan como actores estratégicos entendidos como individuos, organizaciones o grupos que están en capacidad de apresurar o perturbar la toma de decisiones o la resolución de conflictos colectivos a partir de los recursos que poseen ${ }^{(4)}$. Su capacidad no se limita a aspectos económicos sino también al control de cargos políticos, información, ideas, entre otros ${ }^{(14)}$. Dentro de todo el proceso que se lleva a cabo en los observatorios se encuentra representado cada actor estratégico y sus funciones influyen directamente en la toma de decisiones.

En consecuencia, los observatorios se constituyen en actores clave y, a su vez, son escenario de interacción para la toma de decisiones en salud pública, y permiten resolver los conflictos existentes entre los actores generando cambios positivos en las situaciones que se reconocen como problemáticas por ellos y por la ciudadanía.

Por otro lado, la comunidad también es considerada como actor estratégico, a pesar de que no influye en el proceso de validación de los datos. La percepción de la ciudadanía permite aterrizar las decisiones basadas en evidencia. Los conocimientos de una comunidad sobre cierto sector sirven para ajustar las medidas a las necesidades específicas de los ciudadanos (15). Para lograr la participación activa de la comunidad es necesario que se compartan los resultados obtenidos por los observatorios y las medidas que se pretenden implementar, para así ganar la confianza y construir una red de información que beneficie a las instituciones del Estado y a la comunidad misma ${ }^{(10)}$

Para que exista gobernabilidad no es solo necesaria la existencia de actores estratégicos, sino también una serie de reglas y procedimientos claros que sirvan para tomar decisiones y permitan a los actores resolver sus conflictos.
Las reglas y procedimientos establecidos constituyen el régimen político de un país y la institucionalización de las mismas genera mayor gobernabilidad ${ }^{(4)}$.

Sin embargo, los actores estratégicos no siempre actúan bajo reglas o procedimientos adecuados, debido a que no es una tarea fácil realizar consensos en los entes territoriales ${ }^{(16)}$. Los gobiernos locales evitan expresar de manera clara ciertas decisiones ya que, en algunos casos, implica perder legitimidad y apoyo de ciertos sectores; por esta razón suelen presentarse los planes sobre temas controversiales -como la seguridaden términos vagos, indeterminados o a partir de formulaciones generales, con el fin de lograr el respaldo necesario desde la comunidad y sobre todo desde otros actores estratégicos ${ }^{(16)}$.

\section{FORMULACIÓN Y EVALUACIÓN DE POLÍTICAS PÚBLICAS}

Los tomadores de decisiones deben garantizar que la información que obtienen sea la base de la formulación de medidas, estrategias y políticas públicas. Estas son el conjunto de objetivos que se consideran deseables y necesarios, que a través de instituciones $\mathrm{u}$ organizaciones gubernamentales resuelven, por lo menos de manera parcial, una situación que se percibe como problemática o insatisfactoria ${ }^{(16)}$. Esto se configura como el resultado final del proceso que se realiza en los observatorios.

El sistema político está conformado por inputs (demandas de la sociedad) y outputs (soluciones que da el sistema político). Las acciones de gobierno son las respuestas a las demandas de la sociedad a través de las políticas públicas, buscando modificar una situación problemática o llevarla a niveles manejables ${ }^{(5)}$. Los observatorios aportan a la identificación de situaciones problemáticas y orientan la intervención (solución o manejo) de estas. Las políticas públicas deben ser evaluadas de manera constante para no solo formular nuevas políticas públicas, sino generar cambios incrementales a las que ya existen. Los observatorios permiten la evaluación de intervenciones buscando que se generen las modificaciones necesarias a políticas existentes para hacerlas más efectivas ${ }^{(17)}$.

Teniendo en cuenta que las políticas públicas tienen tres etapas: formulación, implementación y evaluación, el realizar un proceso riguroso a través de las etapas garantiza una intervención efectiva ${ }^{(16)}$. En la formulación de la política se define el problema (identificar cuál es, cuáles son sus dimensiones, sus causas, cómo evoluciona si no se actúa y a quién afecta y cómo) ${ }^{(16)}$. Cuando el problema ha sido identificado se deben encontrar las alternativas para su solución y escoger la más adecuada. En este 
proceso intervienen numerosos actores (políticos, administrativos, sociales, económicos, científicos, académicos) que van a influir en la toma de decisiones a partir de sus recursos e intereses ${ }^{(17)}$. Un modelo que describe la interacción entre la política pública, los actores estratégicos y los observatorios esta descrito en la Figura 1.

La etapa de formulación comienza con un análisis del problema, momento en el cual los observatorios cumplen un papel importante al orientar a los actores en la selección de alternativas para darle solución al problema (o decidir no actuar) ${ }^{(19,20)}$. Durante la formulación de una política pública se busca identificar la alternativa más adecuada que permita disminuir la tensión entre el deber ser (situación deseada) y el ser (situación presente); para esto se deben identificar los fines o metas de la política ${ }^{(21)}$.

Una de las actividades centrales en la formulación de políticas públicas es la planificación (busca alcanzar los objetivos de manera coherente, definiendo prioridades y objetivos a corto, mediano y largo plazo) y para llevarla a cabo es indispensable reducir la incertidumbre. Esto implica conocer la situación previa y construir indicadores (sociales, económicos), lo que se constituye en una tarea fundamental para las administraciones con el fin de permitir el seguimiento de los avances a través del tiempo y el espacio ${ }^{(16)}$
Teniendo en cuenta lo anterior, las decisiones se deben tomar siguiendo un proceso racional o basado en evidencia, donde primero se conoce el problema, se determinan los valores y objetivos, se analizan las soluciones posibles $\mathrm{y}$, finalmente, con toda la información, se escoge una solución ${ }^{(22)}$. Sin embargo, estos procesos no corresponden a la forma en que se llevan a cabo; puesto que en la búsqueda de satisfacer necesidades sin acarrear costos de legitimidad, se pueden realizar varios pasos a la vez e incluso omitir alguno ${ }^{(23)}$. Por esta razón, cuando los actores con poder de decisión no cuentan con la información necesaria para reconocer todas las soluciones posibles y escoger la más adecuada; o por el contrario, cuentan con toda la información pero la solución más óptima no responde a sus intereses personales o grupales como institución, proponen soluciones para resolver asuntos inmediatos sin tomar riesgos ${ }^{(24)}$.

Lo anterior responde a un modelo conocido como public choice que pretende estudiar el comportamiento del Estado basándose en el modelo homo economicus, según el cual el individuo actúa de forma egoísta y sus intereses van ligados a maximizar recursos (poder, reelección, ingresos) ${ }^{25)}$. Los tomadores de decisiones buscan resolver temas que afecten a la comunidad de manera inmediata; por lo tanto, sus soluciones no responden a un proceso de selección idóneo sino a una búsqueda por satisfacer de manera temporal lo que se presenta como una situación problemática, con

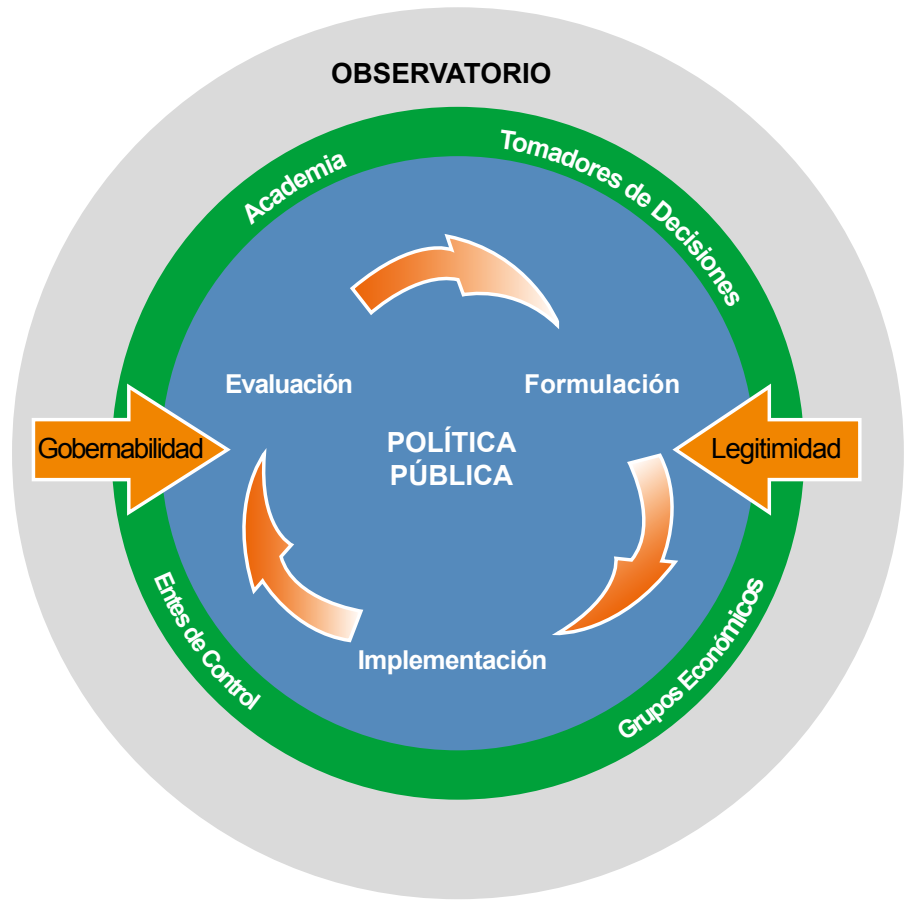

Figura 1. Modelo de interacción entre la política pública, los actores estratégicos y los observatorios para la gobernabilidad 
el fin de minimizar las divergencias con sus electores y asegurar los votos para la siguiente elección, siempre y cuando no se afecte a ningún grupo (económico, político) de su interés ${ }^{(25)}$.

Sin embargo, no se puede olvidar que en las democracias la forma de tomar las decisiones es la negociación -entre actores estratégicos con intereses específicos-, el ajuste entre intereses y la aceptabilidad social, más que la estricta optimización técnico-económica ${ }^{(26)}$, es decir, que no basta con identificar la solución más óptima a partir de la información si no existe un escenario de encuentro de los diferentes intereses como lo son los observatorios y, más específicamente, el espacio brindado por el comité de política social.

La evaluación es una de las etapas más importantes en el análisis de las políticas públicas, en donde se recolecta, verifica e interpreta la información obtenida sobre la implementación y eficacia de políticas públicas y programas públicos ${ }^{(27)}$. Se constituye en una herramienta de los gobiernos democráticos para comprender los procesos sociales y fundamentar acciones futuras, conseguir el respaldo de sus acciones (legitimación) y ejercer control sobre las actividades que realizan ${ }^{(28)}$. Otras definiciones se refieren a la evaluación como una forma de conocer y cuantificar los efectos de una política ${ }^{(29)}$. Por esta razón, contar con un espacio que permita realizar esta actividad de forma segura y eficaz se convierte en una necesidad para los gobiernos.

Uno de los puntos clave y a la vez problemáticos de la evaluación es que se espera que sus resultados respondan a las necesidades de la sociedad y que otorguen legitimidad -basada en el logro de resultados- a las acciones estatales tomando en cuenta eficacia y eficiencia ${ }^{(30)}$.

Teniendo en cuenta los tres tipos de evaluación que existen: ex-ante, concomitante y ex-post, los observatorios participan en las dos últimas. La evaluación concomitante es una evaluación continua que suministra información que permite ajustar los procesos de una acción pública o una evaluación periódica que consiste en el control de las actividades realizadas y permite conocer el estado del proceso teniendo en cuenta lo planeado y establecer los correctivos ${ }^{(16)}$. Para una política pública, este tipo de evaluación en la etapa de implementación permite, a partir de la información que se obtiene de fuentes como los observatorios, la realización de ajustes necesarios para lograr una mayor efectividad de la política sin necesidad de esperar a que se haya implementado en su totalidad y la situación problemática se vea incrementada o no solucionada.

La evaluación ex-post busca analizar los efectos de un programa o una política pública luego de efectuada su implementación ${ }^{(16)}$. Esta evaluación requiere de la utilización de herramientas metodológicas y análisis de los datos adecuados que permitan observar los efectos (positivos, negativos o ninguno) de una política pública determinada que contribuya a la formulación de futuras políticas. Los observatorios cuentan con la información del antes, durante y después de la implementación de una política pública lo que los constituye como una fuente confiable para realizar este tipo de evaluaciones.

La evaluación también es fuente de legitimación de la acción gubernamental. Una evaluación positiva de una política pública se traduce en confianza y apoyo al gobierno (político y/o económico) de actores estratégicos. Esto hace que los gobiernos se esfuercen por obtener resultados positivos en la evaluación o, al menos, mostrar un avance significativo en sus objetivos que, en ocasiones, son contrarios a los resultados obtenidos durante los procesos de evaluación.

En conclusión, los Estados no se rigen en todo momento por reglas formales y procedimientos determinados para generar políticas públicas; sin embargo, buscan solucionar situaciones problemáticas. Para lograrlo es indispensable que cuenten con información confiable que les permita llegar a un consenso entre los intereses de los diferentes actores y las necesidades de la ciudadanía. Para ello, los observatorios de violencia se convierten en la herramienta más adecuada y cercana a la realidad que permite traducir datos en soluciones efectivas, convirtiéndose en facilitadores de interacción de actores, apoyo para legitimar acciones y prerrequisito para la toma de decisiones.

Cada uno de los elementos anteriormente mencionados realizados de manera coherente, adecuada y eficaz, se convierten en una red que favorece la gobernabilidad, desde la detección de un problema, la formulación de una política pública, su implementación y evaluación, pasando por la obtención de legitimación tanto del proceso como de los resultados y evitando conflictos entre los actores; lo que en consecuencia contribuye al fortalecimiento del Estado y su interacción con la ciudadanía.

Contribuciones de autoría: GSR ha participado en la concepción de la investigación, revisión de la literatura, redacción del manuscrito. FJBE ha participado en la concepción de la investigación, asesoría en la búsqueda de la literatura, revisión crítica del manuscrito. AFL ha participado en la revisión crítica del manuscrito. MIGM ha participado en la revisión crítica del manuscrito. Todos los autores participaron en la aprobación de la versión final del manuscrito.

Fuentes de financiamiento: ninguna.

Conflicto de interés: los autores declaran no tener conflictos de interés. 


\section{REFERENCIAS BIBLIOGRÁFICAS}

1. Organización Mundial de la Salud. Informe Mundial Sobre la Violencia y la Salud. Resumen. Washington, D.C: OMS; 2002.

2. Desmond Arias E, Babinet N, Bergman M, Cruz JM, Frühling $\mathrm{H}$, Garzón JC, et al. Informe Regional de Desarrollo Humano 2013-2014. Seguridad Ciudadana con Rostro Humano: diagnóstico y propuestas para América Latina. In: Centro Regional de Servicios para América Latina y el Caribe, Dirección Regional para América Latina y el Caribe, Programa de las Naciones Unidas para el Desarrollo (PNUD), editors. New York: PNUD; 2013. p. 265.

3. Salama P. Informe sobre la violencia en América Latina. Revista de economía institucional. 2008;10(18): 81-102.

4. Prats i Català J. Gobernabilidad democrática para el desarrollo humano. Marco conceptual y analítico. Revista Instituciones y Desarrollo. 2001;(10):103-48.

5. Easton D. Categorías para el análisis sistemico de la política. In: Batlle A, editor. Diez textos básicos de Ciencia Política. $3^{\mathrm{a}} \mathrm{ed}$. Barcelona: Ariel Ciencia Política; 2007.

6. Cruz CN. La legitimidad como prerrequisito de la gobernabilidad democrática: Sobre la importancia de la legitimación de la acción gubernamental. Desarrollo Humano e Institucional en América Latina [Internet]. 2001 Aug [cited 2015 Mar 09, 2015];27: [7 pág.] Disponible en: http://www.gigapp. org/administrator/components/ com_jresearch/files/publications/ C UATRO_ T P O S JUSTIFICACI.PDF

7. Camou A. Los desafíos de la gobernabilidad. Mexico D.F.: Plaza y Valdés; 2001. p. 338.

8. Weber M. El político y el científico. Buenos Aires: UNSAM; 1998.

9. Mayorga F, Córdova E. Gobernabilidad y Gobernanza en América latina. Ginebra: Working Paper NCCR Norte-Sur IP8; 2007.
10. Gutierrez-Martinez MI, Del Villin RE, Fandiño A, Oliver RL. The evaluation of a surveillance system for violent and non-intentional injury mortality in Colombian cities. Int J Inj Contr Saf Promot. 2007;14(2):77-84.

11. Dammert L, Gutiérrez MI, Martin G, Paternain R, Peña N. ¿Qué observan los que observan el delito?: Pasado, presente y futuro de los observatorios del crimen y la violencia en América Latina y el Caribe. Washinton, D.C.: Inter-American Development Bank; 2014.

12. Nioche JP. Padioleau JG. L'État au concret. In: Politiques et management public. 1983;1(2):107-10.

13. Espinosa del Vallín R, Gutiérrez MI. Guía Metodológica para la Replicación de Observatorios Municipales de Violencia. Cali, Colombia: Instituto CISALVA, Universidad del Valle; 2008.

14. Coppedge M. "El concepto de la gobernabilidad: Modelos positivos y negativos". In: Vega E, editor. Ecuador: Un problema de gobernabilidad. Quito: Corporación de Estudios para el Desarrollo; 1997.

15. Fracasso L. Planificación comunitaria y participación en los procesos de decisión: Categorías de análisis y argumentos. Biblio 3W. Revista Bibliográfica de Geografía y Ciencias Sociales [Internet]. 2000 [cited 2015 Mar 09, 2015]; 5(216):[about 1 p.]: Available from: http://www.ub.edu/ geocrit/b3w-216.htm

16. Deubel-Roth AN. Políticas públicas: Formulación, implementación y evaluación $1^{\text {a }}$ ed. Bogotá, D.C.: Ediciones Auroras; 2002.

17. Lindblom CE. El proceso de elaboración de políticas públicas. Madrid: Ministerio para las Administraciones Públicas; 1991.

18. Chevalier J, Lochak D. Rationalité juridique et rationalité managériale dans l'administration française. Revue Française d'Administration Publique. 1982;(24):21-33.

19. Bachrach P, Baratz M. Decisions and Nondecisions: An Analytical Framework.
American Political Science Review. 1963;57(3):632-42. doi: http://dx.doi. org/10.2307/1952568

20. Meny I, Thoenig JC. Las políticas públicas. Barcelona: Ariel; 1992.

21. Centre d'étude dtedélC. Cours de lógistique. Geneva: Faculté de Droit, Université de Genéve; 2000.

22. Taylor FW. The Principles of Scientific Management. New York: Cosimo, Incorporated; 2010.

23. Wiltshire K. Scientists and Policymakers: Towards a New Partnership. Int Soc Sci J. 2001;53(170):621-35. doi: 10.1111/1468-2451.00349

24. Aguilar CR, Lima MA. ¿Qué son y para qué sirven las políticas públicas. Contribuciones a las ciencias sociales [Internet]. 2009 Sep [cited 2015 Mar 09, 2015];5:[about 1 p.]: Available from: http://www.eumed.net/rev/ cccss/05/aalf.htm

25. Downs A. An economic theory of political action in a democracy. Jf Political Econ. 1957;65(2):135-50.

26. Monnier E. Evaluation de l'action des pouvoirs publics. Paris: Economica; 1992.

27. Majone G. Evidencia, argumentación y persuasión en la formulación de políticas. Mexico: Colegio Nacional de Ciencias Políticas y Administración Pública, A.C., Fondo de Cultura Económica; 2005.

28. Duran P. Penser l'action publique. Paris: LGDJ; 1999.

29. Corcuff P. Un OVNI dans le pausage français? Eléments de réflexion sur l'evaluation des politiques publiques en France. In Politix. 1993;6(24):190-209.

30. Muller P. Les politiques publiques. Paris: Presses Universitaires de France; 2009.

Correspondencia: Francisco Javier BonillaEscobar,

Dirección: Calle 4B\#36-00, Edificio 100, Oficina 114, Instituto Cisalva, Universidad del Valle, Cali, Colombia.

Teléfono: (+57-2) 5570276

Correoelectrónico: cisalva@correounivalle.edu.co 